\title{
Could any graph be turned into a
} small-world?

Philippe Duchon

Nicolas Hanusse

Emmanuelle Lebhar

Nicolas Schabanel

\section{École Normale Supérieure de Lyon}

46 Allée d'Italie, 69364 Lyon Cedex 07, France

Téléphone : +33(0)4.72.72.80.37

Télécopieur : $+33(0) 4.72 .72 .80 .80$

Adresse électronique : lip@ens-lyon.fr
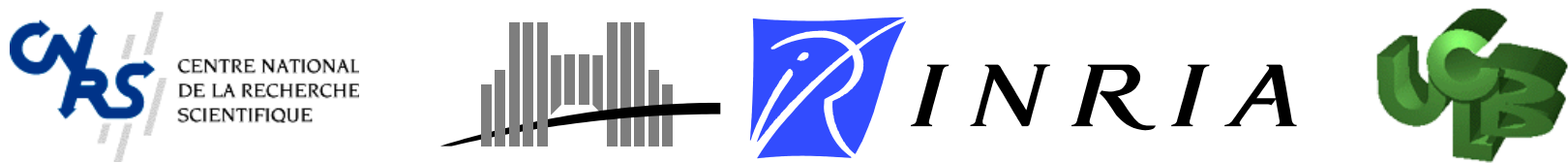


\title{
Could any graph be turned into a small-world?
}

\author{
Philippe Duchon \\ Nicolas Hanusse \\ Emmanuelle Lebhar \\ Nicolas Schabanel
}

Décembre 2004

\begin{abstract}
In addition to statistical graph properties (diameter, degree, clustering, ...), Kleinberg [6] showed that a small-world can also be seen as a graph in which the routing task can be efficiently and easily done. More precisely, in a lattice network augmented by extra random edges (but not chosen uniformly), a short path of polylogarithmic expected length can be found using a greedy algorithm with a local knowledge of the nodes. We call such a graph a navigable smallworld since short paths exist and can be followed with partial knowledge of the network. In this paper, we show that a wide class of graphs can be augmented into navigable small-worlds.
\end{abstract}

Keywords: small-world, random graph model, routing algorithm.

\section{Résumé}

Outre des propriétés statistiques de graphes (diamètre, degré, connectivité, ...), Kleinberg [6] a montré qu'un petit monde peut aussi être considéré comme un graphe où le routage se fait aisément, et de façon efficace. Plus précisément, dans une grille de dimension 2 augmentée par des longs liens aléatoires supplémentaires (mais qui ne sont pas choisis uniformément), l'algorithme glouton trivial trouve un chemin court de longueur moyenne polylogarithmique en utilisant uniquement une connaissance locale des noeuds. Nous appelons un tel graphe petit monde navigable, puisque des chemins courts existent et peuvent être suivis en utilisant une connaissance partielle du réseau. Dans cet article, nous montrons qu'il existe une classe importante de graphes pouvant être augmentés en petits mondes navigables.

Mots-clés: petit monde, modèle aléatoire de graphe, algorithme de routage. 


\section{Introduction}

In the last decade, effective measurements of real interaction networks have revealed specific unexpected properties. Among these, most of these networks present a very small diameter and a high clustering. Furthermore, very short paths can be found efficiently between any pair of nodes without global knowledge of the network, which is known as the small-world phenomenon (exhibited by Milgram [10]). Several models have been proposed to explain this phenomenon. Among them, one approach is based upon an augmentation process : starting from a graph $H$ and adding a relatively small set of extra edges $L$, we hope to obtain a new graph $G$ sharing some graph properties with $H$, and exhibiting additional properties due to the design of $L$. For instance, the circulant graph $H$ consisting in an $n$-node ring where each node is also connected to the $k$-th closest nodes is locally clustered and is often used as a base graph for small-worlds [11,6]. Watts and Strogatz, in 1998 [12], showed that adding a controlled amount of randomness in the circulant graph gives rise to some of the small-world characteristics : rewiring an increasing fraction $\alpha$ of the links to random nodes chosen uniformly exhibits the desired characteristics (locally clustered and small diameter) for a reasonably large range of $\alpha$ away from 0 and 1 . In fact, this model can be easily seen as an augmentation process : allowing to rewire only one link per node (defining in this way $L$ ) would not alter a lot the model and the results (cf. [11]).

However, Kleinberg showed in 2000 [6] that this model lacks the essential navigability property : in spite of a polylogarithmic diameter, none of the short paths can be computed efficiently without global knowledge of the network ; i.e., routing requires the visit of a polynomial number of nodes (in the size of the network). He showed that navigability can be obtained by adjusting the amount of randomness to the underlying metric (the ring here). Precisely, he introduced an augmented graph model consisting of a grid where each node is given a constant number of random additional directed long range links distributed according to the harmonic distribution, i.e., the probability that a node $\mathbf{v}$ is the $i$-th long range contact of a node $\mathbf{u}$ is proportional to $1 /|\mathbf{u}-\mathbf{v}|^{s}$, where $|\mathbf{u}-\mathbf{v}|$ denotes their Manhattan distance in the grid and $s$ is some parameter of the model. In this model, the local knowledge at each node is the underlying metric of the grid (which can be viewed as the geographic locations of the nodes) and the positions on the grid of its long range neighbors. Note that a global knowledge would be the set of positions of all the long range neighbors on the grid. Kleinberg proved that there exists a decentralized algorithm (using only local knowledge) that computes, between any pair of nodes, a path of polylogarithmic length in the size of the network, after visiting a polylogarithmic number of nodes, if and only if the exponent $s$ is equal to the dimension of the grid. The simplest such algorithm is greedy routing : each node obliviously forwards the message to its neighbor (local or long range) that is the closest (in the known metric) to the destination. Later on, Barrière et al. [1] generalized this result to a torus of any dimension. Moreover, they showed that the expected number of steps of the greedy algorithm is $\Theta\left(\log ^{2} n\right)$, and that, noticeably, the number of steps is independent of the dimension. This reveals a strong correlation between the underlying grid metric and the additional long range links distribution that turns the grid into a small-world. This statement raises an essential question to capture the small-world phenomenon : are there only specific graph metrics that can be turned into small-worlds by the addition of shortcuts?

This impression can be reinforced by the fact that whenever the exponent $s$ is different from the dimension of the grid, the greedy algorithm follows a path of polynomial length even when the diameter is polylogarithmic. For Kleinberg's model, Martel and Nguyen [9] proved that the diameter is $\Theta(\log n)$ for $s=d$ and it is conjectured that for $s<2 d$, it is polylogarithmic. For a slightly different percolation model (in which degrees are unbounded), Coppersmith et al. [3] showed indeed a polylogarithmic upper bound on the diameter whenever $s<2 d$. The reader might believe that the navigability property is very specific to the grid topology, but we will show that a wide family of graphs can be turned into navigable small-worlds.

In section 2, we give a formal definition of navigable small-world graphs with respect to a given underlying metric. In this paper, we attempt to find a class of graph metrics as wide as possible for which the addition of random long range links gives rise to the small-world phenomenon. Roughly speaking, as soon as the original graph $H$ is homogeneous in terms of ball expansion and as soon as balls centered on each node grow up to slightly more than polynomially with their radius, $H$ can be augmented to become a navigable small-world. It follows that a wide class of graphs can be turned into a navigable small-world, in particular including known Cayley graphs. In a second step, we try to catch the dimensional phenomenon by studying the cartesian product of our graphs. We show that if two independent graphs can be augmented into two 
navigable small-worlds then their cartesian product can also be augmented into a navigable small-world. For instance, as a consequence, any unbalanced torus $C_{n_{1}} \times C_{n_{2}} \times \ldots \times C_{n_{l}}$ can be turned into a small-world in which the greedy algorithm computes paths of length $O\left(\log ^{2+\epsilon}\left(\max _{i} n_{i}\right)\right)$, for any $\epsilon>0$.

\section{Small-worlds and graph metrics}

For a given graph $G=(V, E)$, we write $\mathcal{B}_{G, \mathbf{u}}(r)$ for the ball centered on a node $\mathbf{u}$ with radius $r$, and $b_{G, \mathbf{u}}(r)$ for its cardinality. Let $b_{G}(r)=\max _{\mathbf{u} \in V} b_{G, \mathbf{u}}(r)$. The $G$ subscript will be omitted in case the concerned graph is obvious. We only consider graphs with maximum degree $\Delta$, a fixed constant.

In the following, an underlying metric $\delta_{H}$ of a graph $G$ is the metric given by a spanning connected subgraph $H$ (i.e., $\delta_{H}(\mathbf{u}, \mathbf{v})$ is the distance between $\mathbf{u}$ and $\mathbf{v}$ in $H$ ). Definitions 1 and 2 are inspired by the work of Kleinberg [6].

Definition $1 \mathrm{~A}$ decentralized algorithm using an underlying metric $\delta_{H}$ in a graph $G$ is an algorithm that computes a path between any pair of nodes by navigating through the network from the source to the target, using only the knowledge 1) of $\delta_{H}$ 2) of the nodes it has previously visited as well as their neighbors. But, crucially, 3) it can only visit nodes that are neighbors of previously visited nodes.

The efficiency of a decentralized algorithm depends crucially on the number of nodes it visits to compute a path. Note that it upper bounds the length of the computed path.

Our definition of a navigable small-world is essentially probabilistic. We consider random graph models in which a fixed "base" graph $H$ is randomly augmented by adding random links (called long links below), according to some probability distribution. Routing will then be performed by a decentralized algorithm, using the base metric $\delta_{H}$ (which is obviously an upper bound on distances in the augmented graph); our goal is to identify such augmented graph models for which this procedure results in uniformly "fast" routing.

Given an underlying metric $\delta_{H}$ in a graph $G$, the maximum length of shortest paths one can hope to find in a constant degree graph $G$ to reach all the nodes at distance $r$ in $H$ from a source $\mathbf{u}$, is lower bounded by $\Omega\left(\log b_{H, \mathbf{u}}(r)\right)$. This motivates the following definition.

Definition 2 An infinite randomly augmented graph $G$, with base graph $H$, is a navigable small-world if there exists a decentralized algorithm using the underlying base metric $\delta_{H}$ that, for any two nodes $\mathbf{u}$ and $\mathbf{v}$, computes a path from $\mathbf{u}$ to $\mathbf{v}$ in $G$ by visiting an expected number of nodes that is polylogarithmic in $b_{H, \mathbf{v}}\left(\delta_{H}(\mathbf{u}, \mathbf{v})\right)$.

A family of finite randomly augmented graphs $\left(G_{i}\right)_{i \in \mathcal{I}}$, with base graphs $H_{i}$ for each $G_{i}$, is a navigable small-world family if there exists a (uniform) polynomial p, and a decentralized algorithm using the underlying base metric $\delta_{H_{i}}$ on $G_{i}$ that, for every $i \in \mathcal{I}$ and any pair of nodes $\mathbf{u}$ and $\mathbf{v}$ in $G_{i}$, computes a path from $\mathbf{u}$ to $\mathbf{v}$, by visiting an expected number of nodes at most $p\left(\log b_{H_{i}, \mathbf{v}}\left(\delta_{H_{i}}(\mathbf{u}, \mathbf{v})\right)\right)$.

Note that some graphs are intrinsically navigable small-worlds (and do not require any augmentation). Indeed, any graph such that each $b_{\mathbf{v}}(r)$ is bounded below by a (uniform) exponential function of $r$ is a smallworld, since the greedy algorithm, using the graph metric, computes a path of optimal length $r=\delta(\mathbf{u}, \mathbf{v})$ between two nodes at distance $r$, and $r=\operatorname{polylog}(b(r))$ in this case. The simplest example of this situation is an infinite $k$-ary tree (with $k \geq 3$ ); balls with radius $r$ have size $\Theta\left((k-1)^{r}\right)$.

\section{$3 \quad$ Turning graphs into small-worlds}

In this section, we describe a wide class of infinite graphs, or of infinite families of finite graphs, for which we are able to define random augmentation models that result in navigable small-worlds. In all cases, our routing algorithm will be the greedy algorithm, thus the set of visited nodes will coincide with the path computed. Furthermore, even if some algorithms can compute significantly shorter paths $[4,9,7]$, it has been shown in [8] that no decentralized algorithm can compute a polylogarithmic path between two nodes while visiting significantly less nodes than the greedy. 
All models we will consider add exactly one directed edge ${ }^{1}$ leaving each node $\mathbf{u}$, and the destination $L_{\mathbf{u}}$ of this outgoing edge is randomly chosen according to a random distribution that gives equal weight to any two nodes that are equally distant from $\mathbf{u}$ (in the base graph). Thus, for each node $\mathbf{u}$, there is a function $f_{\mathbf{u}}$ such that each other node $\mathbf{v}$ has probability proportional to $f_{\mathbf{u}}(\delta(\mathbf{u}, \mathbf{v}))$ of being $L_{\mathbf{u}}$; the normalizing factor $Z_{\mathbf{u}}$ is

$$
Z_{\mathbf{u}}=\sum_{\mathbf{v} \in V} f_{\mathbf{u}}(\delta(\mathbf{u}, \mathbf{v}))=\sum_{r>0}\left(b_{\mathbf{u}}(r)-b_{\mathbf{u}}(r-1)\right) f_{\mathbf{u}}(r) .
$$

Definition 3 We say that an infinite graph is smallworldizable if there exists, for each $\mathbf{u}$, a distribution $f_{\mathbf{u}}(r)$ such that the randomly augmented graph obtained by the addition of one random long range link to each node $\mathbf{u}$ according to $f_{\mathbf{u}}(r)$ (any node $\mathbf{u}$ is the origin of one long range link whose destination is $\mathbf{v}$ with probability proportional to $f_{\mathbf{u}}(\delta(\mathbf{u}, \mathbf{v}))$, is a navigable small-world.

Similarly, we say that an infinite family of finite graphs $\left(H_{i}\right)_{i \in \mathcal{I}}$ is smallworldizable if there exists, for each $\mathbf{u}$ and $i$, a distribution $f_{i, \mathbf{u}}(r)$, such that the family of finite randomly augmented graphs $\left(G_{i}\right)_{i \in \mathcal{I}}$, where $G_{i}$ is obtained by the addition of one random long range link to each node $\mathbf{u}$ of $H_{i}$ according to $f_{i, \mathbf{u}}(r)$ (any node $\mathbf{u}$ is the origin of one long range link whose destination is $\mathbf{v}$ with probability proportional to $f_{i, \mathbf{u}}\left(\delta_{H_{i}}(\mathbf{u}, \mathbf{v})\right)$, is a navigable small-world family.

The following class of graph is defined for the sake of readability. As shown below, it characterizes a class of smallworldizable graphs.

Definition 4 A bounded degree infinite graph $H$ is a moderate growth graph if there exists a constant $\alpha>0$, such that the ball size of each node $\mathbf{u}$ of $H$ can be written as $b_{\mathbf{u}}(r)=r^{d_{\mathbf{u}}(r)}$, where $d_{\mathbf{u}}(r):[2, \infty) \rightarrow \mathbb{R}$ is $\mathcal{C}^{1}$ and satisfies $\forall r \geq 2, d_{\mathbf{u}}^{\prime}(r) \leq \alpha /(r \ln r)$. Similarly, an infinite family of finite uniformly bounded degree graphs $\left(H_{i}\right)_{i \in \mathcal{I}}$ is a moderate growth graphs family if there exists a uniform constant $\alpha>0$, such that the ball size of each node $\mathbf{u}$ of each $H_{i}$ can be written as $b_{i, \mathbf{u}}(r)=r^{d_{i, \mathbf{u}}(r)}$, where $d_{i, \mathbf{u}}(r):[2, \infty) \rightarrow \mathbb{R}$ is $\mathcal{C}^{1}$ and satisfies $\forall r \geq 2, d_{i, \mathbf{u}}^{\prime}(r) \leq \alpha /(r \ln r)$.

Note that the function $d_{\mathbf{u}}(r)$ is simply defined as a $\mathcal{C}^{1}$ interpolation of $\left(\ln \left(b_{\mathbf{u}}(r)\right) / \ln r\right)_{r \in\{2,3, \ldots\}}$, then the $\mathcal{C}^{1}$ condition is not restrictive by itself. We can now state our main result.

Theorem 1 Any moderate growth infinite graph is smallworldizable by the addition of one long range link per node, distributed according to $f_{\mathbf{u}}(r)=\frac{1}{b_{\mathbf{u}}(r) \log ^{q} r}$, for any $q>1$. Any infinite moderate growth graphs family $\left(H_{i}\right)_{i \in \mathcal{I}}$ is smallworldizable by the addition of one long range link per node, distributed according to $f_{i, \mathbf{u}}(r)=\frac{1}{b_{H_{i}, \mathbf{u}}(r) \log ^{q} r}$ in each graph $H_{i}$, for any $q>1$.

Proof. We consider the greedy routing algorithm that, at each step, forwards the message to the closest (in the sense of the $\delta_{H}$ metric) neighbor (in the augmented graph) of the current node. Assume that $\mathbf{s}$ and $\mathbf{t}$ are respectively the source and target. The main argument in Kleinberg's analysis, from which our proof is inspired, is that, among a polylogarithmic number of nodes at distance between $r$ and $r / 2(r \geq 2)$ of the target, with constant probability, at least one node has a long range link that goes to a node at distance less than $r / 2$ from the target, which gives the polylogarithmic path length. We use a similar argument, modified so that the upper bound can be expressed only in terms of the original metric (and not the total size of the graph).

Note that, in order to show that the family $\left(H_{i}\right)_{i \in \mathcal{I}}$ is smallworldizable, we only need to obtain uniform bounds (independent of $i$ ) on the expected path length computed by the greedy algorithm on any finite graph $H_{i}$. Since the proof is analogous for finite and infinite graphs, we will only focus on the infinite graph case.

We first need to check that the normalization constants $Z_{\mathbf{u}}=\sum_{\mathbf{v} \in V} f_{\mathbf{u}}\left(\delta_{H}(\mathbf{u}, \mathbf{v})\right)$ are uniformly bounded (with respect to $\mathbf{u}$ and $i$ ), so that the distribution is properly defined. Let $\alpha>0$ such that $d_{\mathbf{u}}^{\prime}(r) \leq \alpha /(r \ln r)$. Since the maximum degree is bounded by $\Delta$, integrating the upper bound on $d_{\mathbf{u}}^{\prime}$ yields a uniform upper bound on $d_{\mathbf{u}}(r)$. There exists a constant $C>0$ such that, for any $\mathbf{u}$ and $r>1$,

$$
d_{\mathbf{u}}(r) \leq C+\alpha \ln \ln r .
$$

\footnotetext{
${ }^{1}$ Adding a constant number $k$ of edges instead of one would not significantly alter the results, as will be made clear by the proofs.
} 
This, in turn, implies that all normalization constants $Z_{\mathbf{u}}$ are uniformly bounded. Indeed, we have

$$
Z_{\mathbf{u}}=\sum_{r \geq 1}\left(b_{\mathbf{u}}(r)-b_{\mathbf{u}}(r-1)\right) f_{\mathbf{u}}(r)
$$

and the upper bound on $d_{\mathbf{u}}^{\prime}$ implies, for $b_{\mathbf{u}}(r)=\exp \left(d_{\mathbf{u}}(r) \ln r\right)$ :

$$
b_{\mathbf{u}}(r)-b_{\mathbf{u}}(r-1) \leq\left(\frac{1}{r-1} \max d_{\mathbf{u}}(t)+\ln (r) \max d_{\mathbf{u}}^{\prime}(t)\right) b_{\mathbf{u}}(r),
$$

where both maxima are over $t \in[r-1, r]$. Using the previous upper bounds on $d_{\mathbf{u}}$ and $d_{\mathbf{u}}^{\prime}$, we get

$$
\left(b_{\mathbf{u}}(r)-b_{\mathbf{u}}(r-1)\right) f_{\mathbf{u}}(r) \leq\left(\frac{C+\alpha \ln \ln (r)}{r-1}+\frac{\alpha \ln (r)}{(r-1) \ln (r-1)}\right) \frac{1}{\ln ^{q}(r)}
$$

and the sum over $r$ of the right-hand side converges to some constant $Z<\infty$.

One can bound the ratio $b_{\mathbf{u}}(r) / b_{\mathbf{u}}(\beta r)$, for any $0<\beta<1$, as follows :

$$
b_{\mathbf{u}}^{\prime}(r)=\left(d_{\mathbf{u}}^{\prime}(r) \ln r+\frac{d_{\mathbf{u}}(r)}{r}\right) \quad b_{\mathbf{u}}(r) \leq \frac{C+\alpha+\alpha \ln \ln r}{r} b_{\mathbf{u}}(r)
$$

Integrating the ratio $b_{\mathbf{u}}^{\prime}(r) / b_{\mathbf{u}}(r)$ between $\beta r$ and $r$ gives :

$$
\ln \left(\frac{b_{\mathbf{u}}(r)}{b_{\mathbf{u}}(\beta r)}\right) \leq-C \ln \beta+\alpha \ln r \ln \ln r-\alpha \ln (\beta r) \ln \ln (\beta r) .
$$

But, $\ln \ln (\beta r)=\ln \ln r+\ln \left(1+\frac{\ln \beta}{\ln r}\right) \geq \ln \ln r+C^{\prime} \frac{\ln \beta}{\ln r}$ for some constant $C^{\prime}>0$, by concavity of $\ln$. Thus,

$$
\ln \left(\frac{b_{\mathbf{u}}(r)}{b_{\mathbf{u}}(\beta r)}\right) \leq-\alpha \ln \beta \ln \ln r-\left(C+C^{\prime} \alpha\right) \ln \beta .
$$

We conclude that, for all $\mathbf{u}, r \geq 2$ and $0<\beta<1$,

$$
b_{\mathbf{u}}(r) \leq \frac{(\ln r)^{-\alpha \ln \beta}}{\beta^{C+C^{\prime} \alpha}} b_{\mathbf{u}}(\beta r) .
$$

We now analyze the expected path length computed by the greedy algorithm. Consider some integer $r \geq 2$ and a node $\mathbf{u}$ such that $r / 2<\delta_{H}(\mathbf{u}, \mathbf{t}) \leq r$, and denote by $L_{\mathbf{u}}$ the destination of the long range link from u. We give a lower bound on $\mathbb{P}\left[\delta_{H}\left(L_{\mathbf{u}}, \mathbf{t}\right) \leq r / 2\right]$, the probability that the destination node $L_{\mathbf{u}}$ belongs to $\mathcal{B}_{\mathbf{t}}(r / 2)$. Since $f_{\mathbf{u}}$ is a decreasing function and $\mathcal{B}_{\mathbf{t}}(r / 2) \subseteq \mathcal{B}_{\mathbf{u}}(3 r / 2)$, each node of $\mathcal{B}_{\mathbf{t}}(r / 2)$ has probability at least $f_{\mathbf{u}}(3 r / 2) / Z$ of being $L_{u}$. Since, in turn, $\mathcal{B}_{\mathbf{u}}(3 r / 2) \subseteq \mathcal{B}_{\mathbf{t}}(5 r / 2)$, we can give a lower bound on $f_{\mathbf{u}}(3 r / 2)$ in terms of $b_{\mathbf{t}}$ :

$$
f_{\mathbf{u}}(3 r / 2) \geq \frac{1}{b_{\mathbf{t}}(5 r / 2) \ln ^{q}(3 r / 2)} .
$$

Thus, we get a lower bound, depending only on $\mathbf{t}$ and $r$, on the wanted probability :

$$
\begin{aligned}
\mathbb{P}\left[\delta_{H}\left(L_{\mathbf{u}}, \mathbf{t}\right) \leq r / 2\right] & \geq \frac{1}{Z \ln ^{q}(3 r / 2)} \frac{b_{\mathbf{t}}(r / 2)}{b_{\mathbf{t}}(5 r / 2)} \\
& \geq\left(Z 5^{C+C^{\prime} \alpha} \ln ^{q}(3 r / 2) \ln ^{\alpha \ln 5}(5 r / 2)\right)^{-1} \\
& \geq\left(Z 2^{q+\alpha \ln 5} 5^{C+C^{\prime} \alpha} \ln ^{q+\alpha \ln 5}(r)\right)^{-1}
\end{aligned}
$$

We now turn back to the initial question of the length of the greedy path from $\mathbf{s}$ to $\mathbf{t}$. We mentally partition the whole graph into concentric shells centered on $\mathbf{t}$, where the $k$-th shell consists of all nodes whose $\delta_{H}$ distance to $\mathbf{t}$ is between $2^{k-1}$ and $2^{k}$. The previous discussion proves that each node in the $k$-th shell has probability $\Omega\left(k^{-\gamma}\right)$ of having its long range contact in some $i$-th shell with $i<k$, where $\gamma=q+\alpha \ln 5$. 
Thus, the greedy algorithm, once it reaches the $k$-th shell, examines at most $O\left(k^{\gamma}\right)$ vertices on expectation before it finds one vertex whose long range link leads into a smaller shell. By linearity of expectation, the expected length of the greedy path from a vertex in the $k$-th shell is $O\left(k^{1+\gamma}\right)$, with (uniform) constants that can be recovered from the above discussion. As a result, the expected length of the greedy path from $\mathbf{s}$ to $\mathbf{t}$ is polylogarithmic in $\ell=\delta_{H}(\mathbf{s}, \mathbf{t})\left(O\left(\ln ^{1+q+\alpha \ln 5} \ell\right)\right)$, and a fortiori in $b_{\mathbf{t}}\left(\delta_{H}(\mathbf{s}, \mathbf{t})\right)$. Thus, the augmented graph is a randomized navigable small-world.

This theorem covers graphs with ball sizes $b(r)$ growing like $r^{\alpha \log \log r}, \alpha>0$, or slower. Note that we get a similar upper bound $O\left(\ln ^{2+\epsilon} r\right)$, for any $\epsilon>0$, on the expected length of the greedy path between any pair of nodes at distance $r$ from each other. In a vertex-transitive graphs, all balls grow similarly, since for any pair of nodes $(\mathbf{u}, \mathbf{v}), b_{\mathbf{u}}(r)=b_{\mathbf{v}}(r)$ for any radius $r$. Remind that a graph is vertex-transitive iff for all pair of nodes $(\mathbf{u}, \mathbf{v})$, there exists a one-to-one function $\sigma$ on the vertices preserving the edges, such that $\sigma(\mathbf{u})=\mathbf{v}$. Among these graphs, all known Cayley graphs ${ }^{2}$ are smallwordizable : either they are covered by our theorem (polynomial expansion means $\alpha=0$ ) or the diameter is polylogarithmic (exponential or almost exponential expansion, i.e. $\Omega\left(2^{a r^{b}}\right)$ for some $\left.a, b>0\right)$. Indeed, groups of intermediate ball size, between polynomial and exponential, are still unknown, and it is an open question whether there exists a group with ball size $b(r)$ superpolynomial but less than $e^{\sqrt{r}}$, see for instance [5] and [2] for a state of the art.

Product of small-worlds. A remarkable fact on the small-world property is its relative independence of the metric dimension in Kleinberg's model. The expected path length computed by the greedy algorithm is indeed unchanged whether the underlying metric is a ring or a very high dimension grid. This motivates the study of product of smallworldizable graphs.

Definition 5 The cartesian product $H=F \times G$ of two undirected graphs $F$ and $G$ is the graph $\left(V_{H}, E_{H}\right)$ where $V_{H}=V_{F} \times V_{G}$ and $E_{H}=\left\{\left((f, g),\left(f, g^{\prime}\right)\right): g g^{\prime} \in E_{G}, f \in V_{F}\right\} \cup\left\{\left((f, g),\left(f^{\prime}, g\right)\right): g \in V_{G}, f f^{\prime} \in E_{F}\right\}$.

Note that the cartesian product of two graphs of maximum degrees $\Delta_{F}$ and $\Delta_{G}$ is a graph of maximum degree $\Delta_{F}+\Delta_{G}$.

Theorem 2 Let $F$ and $G$ two moderate growth infinite graphs, $\left(\left(F_{i}\right)_{i \in \mathcal{I}}\right.$ and $\left(G_{i}\right)_{i \in \mathcal{I}}$ two infinite moderate growth graphs families). The cartesian product $H=F \times G$ (the family $\left.\left(H_{i}\right)_{i \in \mathcal{I}}=\left(F_{i} \times G_{i}\right)_{i \in \mathcal{I}}\right)$ is smallworldizable by the addition of one long range link per node $\mathbf{u}$ according the distribution $h_{\mathbf{u}}(r)=1 /\left(b_{H, \mathbf{u}}(r) \ln ^{q^{\prime}} r\right)$ $\left(h_{i, \mathbf{u}}(r)=1 /\left(b_{H_{i}, \mathbf{u}}(r) \ln ^{q^{\prime}} r\right)\right.$ for each graph $\left.H_{i}\right)$, for all $q^{\prime}>q_{0}$, for some constant $q_{0}>0$.

Proof. As in proof of Theorem 1, we only prove the result for infinite graphs, other cases follow. Note that it is unclear whether $H$ is a moderate growth graph (i.e., if $\left.\exists \alpha>0, \forall \mathbf{u}, \forall r \geq 2, d_{H, \mathbf{u}}^{\prime}(r) \leq \alpha /(r \ln r)\right)$.

By construction of the graph $H$, for all $\mathbf{u}=\left(\mathbf{u}_{1}, \mathbf{u}_{2}\right)$ in $H, b_{F, \mathbf{u}_{1}}(r / 2) b_{G, \mathbf{u}_{2}}(r / 2) \leq b_{H,\left(\mathbf{u}_{1}, \mathbf{u}_{2}\right)}(r) \leq b_{F, \mathbf{u}_{1}}(r) b_{G, \mathbf{u}_{2}}(r)$. Let $\alpha_{1}$ and $\alpha_{2}$ such that $d_{F, \mathbf{u}_{1}}^{\prime}(r) \leq \alpha_{1} /(r \ln r)$ and $d_{G, \mathbf{u}_{2}}^{\prime}(r) \leq \alpha_{2} /(r \ln r)$, for all $\mathbf{u}_{1}$ in $F$ and $\mathbf{u}_{2}$ in $G$. From Equation (1), there exists a constant $A>0$ such that

$$
\begin{aligned}
b_{F, \mathbf{u}_{1}}(r) b_{G, \mathbf{u}_{2}}(r) & \leq A(\ln r)^{\left(\alpha_{1}+\alpha_{2}\right) \ln 2} b_{F, \mathbf{u}_{1}}(r / 2) b_{G, \mathbf{u}_{2}}(r / 2) \\
& \leq A(\ln r)^{\left(\alpha_{1}+\alpha_{2}\right) \ln 2} b_{H,\left(\mathbf{u}_{1}, \mathbf{u}_{2}\right)}(r)
\end{aligned}
$$

We first need to check that the normalization constants $Z_{\mathbf{u}}=\sum_{\mathbf{v} \in V_{H}} h_{\mathbf{u}}\left(\delta_{H}(\mathbf{u}, \mathbf{v})\right)$ are uniformly bounded,

${ }^{2}$ A Cayley graph is a graph defined by a group $G$ whose vertices are the elements of $G$ and such that there is an edge between $x$ and $y$ iff there is an element $g \in G$ such that $x=g y$. 
so that the distribution is properly defined. For $q^{\prime}>2+\left(\alpha_{1}+\alpha_{2}\right) \ln 2=_{\mathrm{def}} q_{0}$,

$$
\begin{aligned}
Z_{\mathbf{u}} & =\sum_{\mathbf{v} \in V_{H}} \frac{1}{b_{H, \mathbf{u}}\left(\delta_{H}(\mathbf{u}, \mathbf{v})\right) \ln ^{q^{\prime}}\left(\delta_{H}(\mathbf{u}, \mathbf{v})\right)} \\
& \leq \sum_{\mathbf{v} \in V_{H}} \frac{A\left(\ln \left(\delta_{H}(\mathbf{u}, \mathbf{v})\right)\right)^{\left(\alpha_{1}+\alpha_{2}\right) \ln 2}}{b_{F, \mathbf{u}_{1}}\left(\delta_{H}(\mathbf{u}, \mathbf{v})\right) b_{G, \mathbf{u}_{2}}\left(\delta_{H}(\mathbf{u}, \mathbf{v})\right) \ln q^{q^{\prime}}\left(\delta_{H}(\mathbf{u}, \mathbf{v})\right)} \\
& \leq A \sum_{\mathbf{v} \in V_{H}} \frac{\left(\ln \left(\delta_{F}\left(\mathbf{u}_{1}, \mathbf{v}_{1}\right)\right)\right)^{\frac{\left(\alpha_{1}+\alpha_{2}\right) \ln 2-q^{\prime}}{2}}}{b_{F, \mathbf{u}_{1}}\left(\delta_{F}\left(\mathbf{u}_{1}, \mathbf{v}_{1}\right)\right)} \frac{\left(\ln \left(\delta_{G}\left(\mathbf{u}_{2}, \mathbf{v}_{2}\right)\right)\right)^{\frac{\left(\alpha_{1}+\alpha_{2}\right) \ln 2-q^{\prime}}{2}}}{b_{G, \mathbf{u}_{2}}\left(\delta_{G}\left(\mathbf{u}_{2}, \mathbf{v}_{2}\right)\right)} \\
& \leq A\left(\sum_{\mathbf{v}_{1} \in V_{F}} \frac{\left(\ln \left(\delta_{F}\left(\mathbf{u}_{1}, \mathbf{v}_{1}\right)\right)\right)^{\frac{\left(\alpha_{1}+\alpha_{2}\right) \ln 2-q^{\prime}}{2}}}{b_{F, \mathbf{u}_{1}}\left(\delta_{F}\left(\mathbf{u}_{1}, \mathbf{v}_{1}\right)\right)}\right)\left(\sum_{\mathbf{v}_{2} \in V_{G}} \frac{\left(\ln \left(\delta_{G}\left(\mathbf{u}_{2}, \mathbf{v}_{2}\right)\right)\right)^{\frac{\left(\alpha_{1}+\alpha_{2}\right) \ln 2-q^{\prime}}{2}}}{b_{G, \mathbf{u}_{2}}\left(\delta_{G}\left(\mathbf{u}_{2}, \mathbf{v}_{2}\right)\right)}\right) \\
& \leq A Z_{F, \mathbf{u}_{1}} Z_{G, \mathbf{u}_{2}}<A Z_{F} Z_{G}={ }_{\operatorname{def}} Z_{H}<\infty,
\end{aligned}
$$

where $Z_{F, \mathbf{u}_{1}}$ and $Z_{G, \mathbf{u}_{2}}$ are respectively the normalizing constants for nodes $\mathbf{u}_{1}$ in $F$ and $\mathbf{u}_{2}$ in $G$ with $q=\frac{q^{\prime}-\left(\alpha_{1}+\alpha_{2}\right) \ln 2}{2}$, and $Z_{F}$ and $Z_{G}$ are the corresponding uniform bounds given in the proof of Theorem 1 .

As in the proof of Theorem 1 , we lower bound $\mathbb{P}\left[\delta_{H}\left(L_{\mathbf{u}}, \mathbf{t}\right) \leq r / 2\right]$, the probability that the long range contact $L_{\mathbf{u}}$ of a given node $\mathbf{u}$, at distance $r$ to the target $\mathbf{t}$ in $H$, belongs to $\mathcal{B}(\mathbf{t}, r / 2)$.

$$
\begin{aligned}
\mathbb{P}\left[\delta_{H}\left(L_{\mathbf{u}}, \mathbf{t}\right) \leq r / 2\right] & \geq \frac{1}{Z_{H} \ln ^{q^{\prime}}(3 r / 2)} \frac{b_{H, \mathbf{t}}(r / 2)}{b_{H, \mathbf{t}}(5 r / 2)} \\
& \geq \frac{1}{Z_{H} \ln ^{q^{\prime}}(3 r / 2)} \frac{b_{F, \mathbf{t}_{1}}(r / 4) b_{G, \mathbf{t}_{2}}(r / 4)}{b_{F, \mathbf{t}_{1}}(5 r / 2) b_{G, \mathbf{t}_{2}}(5 r / 2)} \\
& \geq\left(Z_{H} 10^{2 C+C^{\prime}\left(\alpha_{1}+\alpha_{2}\right)} \ln ^{q^{\prime}}(3 r / 2) \ln ^{\left(\alpha_{1}+\alpha_{2}\right) \ln 10}(5 r / 2)\right)^{-1} \\
& \geq\left(Z_{H} 2^{q^{\prime}+\left(\alpha_{1}+\alpha_{2}\right) \ln 10} 10^{2 C+C^{\prime}\left(\alpha_{1}+\alpha_{2}\right)} \ln ^{q^{\prime}+\left(\alpha_{1}+\alpha_{2}\right) \ln 10}(r)\right)^{-1} .
\end{aligned}
$$

We conclude as above that the expected path length computed by the greedy algorithm is polylogarithmic in $\delta_{H}(\mathbf{s}, \mathbf{t})\left(O\left(\ln ^{1+q^{\prime}+\left(\alpha_{1}+\alpha_{2}\right) \ln 10}\left(\delta_{H}(\mathbf{s}, \mathbf{t})\right)\right)\right.$ expected length) between $\mathbf{s}$ and $\mathbf{t}$, and then polylogarithmic in $b_{H, \mathbf{t}}\left(\delta_{H}(\mathbf{s}, \mathbf{t})\right) . H$ is then smallworldizable.

Note that this theorem yields another simple method to obtain a generalization of Kleinberg's graph to tori of dimension $d \geq 1$ with arbitrary side sizes, seen as cartesian products of one dimensional Kleinberg graphs of various sizes.

\section{Conclusion and open problems}

In this paper, we extend the scheme introduced by Kleinberg [6], that allows to generate small-worlds from a wide variety of graph topologies. Our scheme treats in particular all known Cayley graphs. We are also able to "smallworldize" less regular graphs whose ball growths depend on their center and their radius and can grow up to $r^{O(\log \log r)}$ for a radius $r$, or that are obtained as product of such graphs (e.g. unbalanced d-dimensional tori).

Theorems 1 and 2 capture wide classes of smallwordizable graphs but leave open the question of determining whether any graph is smallworldizable. The condition on the derivative of the exponent $d_{\mathbf{u}}(r)$ might be too restrictive. If we take a closer look at graphs with polynomial ball expansion, some of them do not satisfy the derivative condition but are still smallworldizable using an augmentation with our distribution. To illustrate this idea, we give an example of an extreme graph containing two distinct parts of different density (or two types of polynomial expansion) connected only by a single node : the infinite fly swatter. This graph is defined by a half infinite chain connected to a half infinite square lattice. This graph has vertex set $\left(\mathbb{Z}_{<0}^{-} \times\{0\}\right) \bigcup\left(\mathbb{Z}_{>0}^{+} \times \mathbb{Z}\right)$ and two nodes are adjacent if the Manhattan distance is equal to one. We give some hints (but do not provide a real proof) on why the augmented infinite fly swatter is a navigable small-world. 
Informally, the chain does not significantly disturb the greedy routing within the half plane. The tough part is to find a short path from $(0,0)$ to $(-n, 0)$. The lattice part is indeed the attractive part of the graph.

Let us consider the current node $\mathbf{u}=(-m, 0)$ and the destination $\mathbf{t}=(-n, 0)$ belonging to the chain with $n>m$. The size of the balls centered on $\mathbf{u}$ are $b_{\mathbf{u}}(r)=2 r+1$ for $r \leq m$ and else $b_{\mathbf{u}}(r)=2 m+(r-$ $m)+(r-m+1)^{2}$. One can check that $d_{\mathbf{u}}(r)=\frac{\ln b_{\mathbf{u}}(r)}{\ln r}$ is close to 1 for $r=m$ and close to 2 for $r=2 m$. It follows that there exists $m \leq r \leq 2 m$ such that $d_{\mathbf{u}}^{\prime}(r)=\Omega(1 / m)$ and that the infinite fly swatter does not fall into the smallwordizable graphs dealt with Theorem $1: d_{u}^{\prime}(t)$ cannot remain lower than $O\left(\frac{\alpha}{r \ln r}\right)$ for $m \in[m, 2 m]$.

However, following the same reasoning as in the proof of Theorem 1, we can first check that the partition functions $Z_{u}$ are uniformly bounded. Moreover, for the destination $\mathbf{t}=(-n, 0)$, with probability $\Omega\left(\log ^{-q} m\right)$, there exists a long-range link leading to a node $\left(-m^{\prime}, 0\right)$ with $m^{\prime} \in[3 m / 2,2 m]$. It follows that after visiting a logarithmic number of nodes, using the greedy algorithm, the message will traverse with constant probability a long-range link and almost double its distance from the origin. This situation occurs roughly $\log n$ times before the message will be very close to $\mathbf{t}$. Of course, others cases occur but they can be reduced to the above discussion.

More generally, other classes of graphs should be smallwordizable : we suspect that smallwordizable graphs could also include subgraphs of $d$-dimensionnal grids or tori. The characterization of smallworldizable graphs is also open whenever the expansion of balls is locally exponential or almost exponential. A reasonable and attractive case is the one of the family of vertex-transitive graphs due to the homegeneity of balls expansion of these graphs.

\section{Références}

[1] L. Barrière, P. Fraigniaud, E. Kranakis, and D. Krizanc. Efficient Routing in Networks with Long Range Contacts. In Proceedings of the 15th International Conference on Distributed Computing (DISC), pages 270-284, 2001.

[2] L. Bartholdi. Groups of intermediate growth, preprint oai :arxiv.org :math/0201293. 2002.

[3] D. Coppersmith, D. Gamarnik, and M. Sviridenko. The diameter of a long-range percolation graph. Random Struct. Algorithms, 21(1):1-13, 2002.

[4] P. Fraigniaud, C. Gavoille, and C. Paul. Eclecticism shrinks even small worlds. In Proceedings of the 23rd ACM Symp. on Principles of Distributed Computing (PODC), 2004.

[5] R.I. Grigorchuk. On growth in group theory. In Proceedings of the International Congress of Mathematicians, Kyoto, pages 325-338, 1990.

[6] J. Kleinberg. The Small-World Phenomenon : An Algorithmic Perspective. In Proceedings of the 32nd ACM Symposium on Theory of Computing (STOC), pages 163-170, 2000.

[7] E. Lebhar and N. Schabanel. Almost optimal decentralized routing in long-range contact networks. In LNCS proceedings of 31st International Colloquium on Automata, Languages and Programming (ICALP), pages 894-905, 2004.

[8] G. S. Manku, M. Naor, and U. Wieder. Know thy neighbor's neighbor : the power of lookahead in randomized $\mathrm{p} 2 \mathrm{p}$ networks. In Proceedings of the 36th ACM Symposium on Theory of Computing (STOC), 2004.

[9] C. Martel and V. Nguyen. Analyzing kleinberg's (and other) small-world models. In Proceedings of the Twenty-Third Annual ACM Symposium on Principles of Distributed Computing, pages 179-188, 2004.

[10] S. Milgram. The small world problem. Psychology Today, 61(1), 1967.

[11] M. E. J. Newman and D. J. Watts. Scaling and percolation in the small-world network model. Phys. Rev., 60 :7332-7342, 1999.

[12] D. Watts and S. Strogatz. Collective dynamics of small-world networks. Nature, 393(440-442), 1998. 